INPLASY

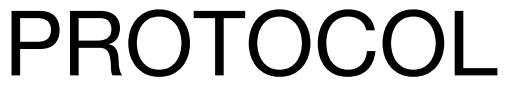

To cite: You et al. Impact of 3D rigid ring Annuloplasty for Tricuspid regurgitation: a systematic review and metaanalysis. Inplasy protocol 202130105. doi:

10.37766/inplasy2021.3.0105

Received: 29 March 2021

Published: 29 March 2021

Corresponding author:

Tao You

youtao2016@126.com

Author Affiliation:

Department of Cardiovascular

Surgery, Gansu Provincial

Hospital

Support: GSWSKY2016-04.

Review Stage at time of this submission: Data extraction.

Conflicts of interest:

None declared.

\section{Impact of 3D rigid ring Annuloplasty for Tricuspid regurgitation: a systematic review and meta-analysis}

You, T1; Ma, YH2; Yi, K³; Zhang, X4; Gao, J5; Xu, XM6; He, SE7; Wang, W8; Ji, M9 .

Review question / Objective: P: Patients with tricuspid regurgitation requiring tricuspid annuloplasty. I/C: Comparison of 3D rigid ring and suture, flexible band, flat rigid ring and other shaping techniques in the treatment of TR. $O$ : aortic cross-clamp (ACC) time, cardiopulmonary bypass (CPB) time, postoperative TR grade (defined as TR grade within one week after surgery), perioperative mortality (defined as hospital mortality or 30-day death rate), late mortality rate (defined as the total mortality rate during follow-up), early complication rate (defined as the rate of complication within 30 days after surgery) and recurrent TR (defined as postoperative moderate and above TR (grade 2-4)). S: Controlled Trial (RCT) or cohort study. Objective: we conducted this systematic review and meta-analysis to compare the effects of 3D rigid annulus and other methods in TAP, and provide a reference for selecting the appropriate annulus type during tricuspid annuloplasty.

INPLASY registration number: This protocol was registered with the International Platform of Registered Systematic Review and Meta-Analysis Protocols (INPLASY) on 29 March 2021 and was last updated on 29 March 2021 (registration number INPLASY202130105).

\section{INTRODUCTION}

Review question / Objective: P: Patients with tricuspid regurgitation requiring tricuspid annuloplasty. I/C: Comparison of 3D rigid ring and suture, flexible band, flat rigid ring and other shaping techniques in the treatment of TR. O: aortic cross-clamp (ACC) time, cardiopulmonary bypass (CPB) time, postoperative TR grade (defined as TR grade within one week after surgery), perioperative mortality (defined as hospital mortality or 30-day death rate), late mortality rate (defined as the total mortality 
rate during follow-up), early complication rate (defined as the rate of complication within 30 days after surgery) and recurrent TR (defined as postoperative moderate and above TR (grade 2-4)). S: Controlled Trial (RCT) or cohort study. Objective: we conducted this systematic review and meta-analysis to compare the effects of 3D rigid annulus and other methods in TAP, and provide a reference for selecting the appropriate annulus type during tricuspid annuloplasty.

Rationale: Tricuspid regurgitation (TR) is a common valvular heart disease (VHD), which occurs in $65 \%$ šC $85 \%$ of the population. A mild TR with a normal structure can be regarded as a normal variant. Moderate to severe TR is usually pathological and is an independent risk factor for progressive heart failure and increased mortality. TR is divided into primary TR and Functional TR (FTR). FTR is caused by the abnormal anatomy and function of the tricuspid valve due to dilation and dysfunction of the right ventricle. At present, FTR is considered to be a continuous process. If it is not treated, disease progression will lead to gradual dilation and dysfunction of the right ventricle, which will seriously affect the prognosis. The AHA and ESC guidelines recommend that patients with severe TR should be treated with the tricuspid valve at the same time as the left heart valve surgery (Class I recommendation). For patients with mild to moderate TR and tricuspid annulus dilation, Tricuspid valve surgery should be considered during the same period of left heart valve surgery (Class Ila recommendation). At present, transcatheter tricuspid valve intervention (TTVI) is developing rapidly, but the technology has not been fully popularized in clinical practice. Tricuspid valve plasty (TVP) is still the main method of surgical treatment of tricuspid regurgitation, mainly including suture annuloplasty and prosthetic tricuspid annuloplasty. Suture annuloplasty, such as the Kay method and De Vega method, has the advantages of simple technology and low patient economic burden, whereas also has a relatively high recurrence and metastasis rate. Compared with suture, prosthetic tricuspid annuloplasty can better prevent annular dilatation, right ventricular volume overload, and right heart failure. Currently, a large number of studies have shown that the ability of prosthetic tricuspid annuloplasty to restore the tricuspid valve is better than suture annuloplasty, so tricuspid annuloplasty (TAP) using various commercially available rings is accepted as the standard technique for correcting TR. According to the rigidity, TAP rings are divided into flexible bands and rigid rings. The former can adapt to the cyclical movement of the heart, while it cannot be maintained for a long time. Long-term right ventricular hypertension and valve movement will gradually expand the annulus and produce regurgitation. The latter is not well adapted to the anatomical characteristics of the tricuspid valve annulus, and the suture will pull the annulus while the maintenance time is relatively long. Due to the unique dynamic three-dimensional (3D) structure of the tricuspid valve, many 3D rigid rings have been developed in recent years. It is believed that the 3D rigid ring can well adapt to the anatomical structure of the tricuspid valve, correct the expansion of the annulus, and prevent further expansion of the annulus. Studies have pointed out that it can enhance the joint force of the valve leaflets, reduce the tension of the suture, and reduce the risk of annulus opening, thereby reducing the possibility of long-term recurrence of tricuspid regurgitation.

Condition being studied: In the past few decades, the surgical results of many types of annuloplasty have been reported clinically, but only a few studies have compared and evaluated these devices. Therefore, It is still inconclusive that which tricuspid annuloplasty ring should be chose in clinical practice. Although the 3D rigid ring has been widely used in clinical practice, there is no relevant research to systematically explain whether it has advantages compared with suture, flexible band and standard rigid ring TAP. Based on this, we conducted this systematic review and meta-analysis to compare the effects 
of $3 D$ rigid annulus and other methods in TAP, and provide a reference for selecting the appropriate annulus type during tricuspid annuloplasty.

\section{METHODS}

Search strategy: \#1 "Tricuspid Valve Insufficiency"[Mesh] \#2 "Tricuspid Valve Insufficiency" \#3 "tricuspid regurgitation" \#4 "Tricuspid Valve Regurgitation" \#5"Tricuspid Valve Incompetence" \#6"Tricuspid Incompetence" \#7"Right atrioventricular valve regurgitation" \#8\#1 OR\#2 OR \#3 OR \#4 OR \#5 OR \#6 OR \#7 \#9"tricuspid valvoplasty" \#10"tricuspid valvuloplasty" \#11"tricuspid annuloplasty" \#12"tricuspid valve prosthesis" \#13"Tricuspid valve plasty" \#14"tricuspid valve repair" \#15"tricuspid valve reconstruction" \#16"tricuspid ring annuloplasty" \#17TVA \#18TAP \#19"Cardiac valve annuloplasty" \#20"Cardiac Valve Annuloplasty"[Mesh] \#21\#9 OR \#10 OR \#11 OR \#12 OR \#13 OR \#14 OR \#15 OR \#16 OR \#17 OR \#18 OR \#19 OR \#20; \#22 \#8 AND \#21.

Participant or population: Patients with tricuspid regurgitation requiring tricuspid annuloplasty.

Intervention: Comparison of 3D rigid ring and suture, flexible band, flat rigid ring and other shaping techniques in the treatment of TR.

Comparator: Comparison of 3D rigid ring and suture, flexible band, flat rigid ring and other shaping techniques in the treatment of TR.

Study designs to be included: Randomized Controlled Trial (RCT) or cohort study.

Eligibility criteria: The inclusion criteria of this study were determined before the literature search. The included studies need to meet the following inclusion criteria: (1) Comparison of 3D rigid ring and suture, flexible band, flat rigid ring and other shaping techniques in the treatment of TR. (2) Randomized Controlled Trial (RCT) or cohort study. (3) If several studies conducted by the same institution have overlapping samples, only the latest research literature will be included.

Information sources: We conducted a systematic literature search on eight databases including PubMed, the Cochrane Library, Web of Science, EMBASE, China National Knowledge Infrastructure (CNKI), China Biology Medicine disc (CBM), Wan Fang and VIP to retrieve all related articles before October 1, 2020. At the same time, we traced the references of the included literature, and found documents through Google Scholar and manual search of related articles. Likewise, we also searched the references of the included literature through the snowball method in order to maximize the sensitivity of retrieval as much as possible.

Main outcome(s): Perioperative mortality (defined as hospital mortality or 30-day death rate), late mortality rate (defined as the total mortality rate during follow-up), early complication rate (defined as the rate of complication within 30 days after surgery), and recurrent TR (defined as postoperative moderate and above TR (grade 2-4)).

Additional outcome(s): Aortic cross-clamp (ACC) time, cardiopulmonary bypass (CPB) time, postoperative TR grade (defined as TR grade within one week after surgery).

Data management: The retrieved articles from the databases were exported to EndNote X8 for duplicate removal and further categorization. The full text of reviews will also be uploaded and attached to EndNote X8. We shall perform predevelopment Microsoft Excel 2019 spreadsheets to extract data and later export into tables and figures.

Quality assessment / Risk of bias analysis: The risk of bias in the included literature was referenced to the Newcastle-Ottawa Scale (NOS). Evaluation items include: (1) Representativeness of the exposed cohort. (2) Selection of the non-exposed cohort. (3) Ascertainment of exposure. (4) Demonstration that outcome of interest 
was not present at start of study. (5) Comparability of cohorts on the basis of the design or analysis. (6) Assessment of outcome. (7) Was follow-up long enough for outcomes to occur. (8) Adequacy of follow up of cohorts. Among them, the fifth item is 2 points, and the remaining 7 are 1 point. The score of the scale is $0-9$, and when the score is $\geq 7$, it is considered to be a study with low risk of bias. The risk deviation assessment was completed by two authors independently, and when differences arose, they were resolved through discussion or negotiated by a third author until agreement was reached.

Strategy of data synthesis: All data analysis was performed using RevMan5.3 software and stata16. We choose unadjusted raw data because various studies have not adjusted for the same set of confounding factors. Binary variables are represented by odds ratio (OR), continuous variables are represented by mean difference (MD) for consistent measurement units, and standardized mean difference (SMD) is used for inconsistent measurement units. All variables are calculated with $95 \%$ confidence intervals $(95 \% \mathrm{Cl})$. All reported $\mathbf{P}$ values are two-sided, and $P 0.10$ and $12 \leq 50 \%$. Otherwise, the heterogeneity of the study was considered significant and the random effects model was used for analysis. Publication bias was assessed by funnel plots and the Egger's test.

Subgroup analysis: If there were identified single factors that influenced heterogeneity between included studies, we would perform a subgroup analysis.

Sensitivity analysis: For studies with significant heterogeneity or high risk of bias, sensitivity analysis will be used to verify the stability of the combined effect.

Language: There will be no restrictions by languages.

Country(ies) involved: China.

Keywords: tricuspid regurgitation; threedimensional rigid ring; tricuspid annuloplasty; meta-analysis; systematic reviews

Dissemination plans: The full article will be published in the public journal as a paper.

Contributions of each author:

Author 1 - Tao You.

Author 2 - Yu-Hu Ma.

Author 3 - Kang Yi.

Author 4 - Xin Zhang.

Author 5 - Jie Gao.

Author 6 - Xiao-Min Xu.

Author 7 - Shao-E He.

Author 8 - Wei Wang.

Author 9 - Meng Ji. 